RHIC $-\mathrm{PH}-8$

OG 882

SOME COMPARISONS OF HIJET WITH DATA

T. Ludlam

February 24, 1986 


\title{
SOME COMPARISONS OF HIJET WITH DATA
}

\author{
Thomas W. Ludlam
}

\section{Introduction}

This note compares HIJET 1.0 with some data samples from $p-p, p-A$ and p-nucleus collisions, with the purpose of trying to gain a comprehensive view of how well the model reproduces well-known features of high energy interactions. We are not trying to break new physics ground here, nor are we trying to make detailed fits (no parameters are adjusted).

\section{Proton-proton Collisions}

Here we are looking strictly at ISAJET, which does the nucleon-nucleon interactions in HIJET. HIJET uses the MINBIAS routine of ISAJET, and uses it at much lower energies than the collider range that ISAJET was designed for and for which it has been extensively tested by high energy users. For HIJET the interesting range of nucleon-nucleon collisions includes laboratory momenta roughly from 1 to $100 \mathrm{GeV} / \mathrm{c}$.

In Fig. 1 we see that the ISAJET charged particle multiplicity agrees very well with data over this momentum range. Figure 2 shows that the higher moments of the multiplicity distributions are also in good agreement with experiment, agreeing well with the empirical kNO scaling curve.

Figure 3 shows the $\mathrm{x}$ distribution for inclusive proton production over the interesting range of energies. The model gives a good accounting of the data except near $x=1.0$, where ISAJET does not include the diffractive peak seen in the data. Figures $4 a$, $b$ show the invariant cross section for proton production as a function of $x$ and $P_{T}$. Again, ISAJET gives good agreement with the data, with the exception of the diffractive peak at $x=1$.

The rapidity spectra for charged particle production are compared with data at $200 \mathrm{GeV} / \mathrm{c}$ in Fig. 5. The data [de Marzo et al., Phys. Lett. 112B, $173(1982)]$ are well reproduced.

Finally, Fig. 6 shows the $E_{T}$ spectrum measured by the NA5 calorimeter at the CERN SPS. This is well reproduced by ISAJET.

We may conclude that the essential dynamics of nucleon-nucleon interactions are very well described by ISAJET, down to the lowest energies of interest for HIJET. The kinematic aspects are done precisely: energy and momentum are rigorously conserved in each collision. The detailed accounting of quantum number flow is not done in ISAJET's MINBIAS routine, except in an average way. Charge and strangeness need not balance exactly in a given event (baryon number will). The $K / \pi$ ratio is roughly $r i g h t$ at high energies 
of interest for HIJET. The kinematic aspects are done precisely: energy and momentum are rigorously conserved in each collision. The detailed accounting of quantum number flow is not done in ISAJET's MINBIAS routine, except in an average way. Charge and strangeness need not balance exactly in a given event (baryon number will). The $K / \pi$ ratio is roughly right at high energies $(\sqrt{52} 50 \mathrm{GeV})$, but there will be too many strange particles to agree with the data at fixed-target energies.

\section{Proton-Nucleus Collisions}

The mean charged-particle multiplicity for proton-nucleus collisions is shown in Fig. 7, for two different energies, as a function of A. Figures 8 and 9 show the charged particle rapidity distributions obtained in the NA5 streamer chamber at the CERN SPS, with argon and xenon, respectively, as the target nuclei [de Marzo et al. Phys. Rev. D26, 1019 (1982)]. HIJET agrees remarkably well with these data. It is this agreement which has been the primary motivation for the widespread use of HIJET as a guide to the expected particle production in the design of high energy nuclear beams experiments.

Figure 10 shows the mean number of collisions per p-nucleus interaction as a function of A. This is somewhat larger in HIJET than the numbers usually extracted from the data (solid line), the effect being greater at small A than at large A. This is due in part to the hard sphere, uniform density distribution employed in HIJET.

An important issue is the so-called "stopping power": the distribution of leading baryons in p-nucleus collisions. In Fig. 11 the HIJET result is compared with the much-studied Fermilab data sample of Barton et al. It will be seen that the agreement is very good for the heavy nuclear targets. For the light nucleus (carbon) the HIJET result is high by about a factor of two in the range of $x$ covered by the data. Note (Fig. 4) that for a proton target the agreement is again very good.

In general, HIJET seems to do very well in reproducing the average values of measured quantities in p-nucleus collisions. It does not do so well in reproducing the fluctuations seen in the data. This is evident in Fig. 12, where the dispersion of the multiplicity distribution is plotted against the mean. The calculated result is in complete agreement with the data for the proton-proton case, but the multiplicity distributions from HIJET are significantly narrower than the data for the heavier nuclei. A similar, and quite possibly related effect is seen in Fig. 13. Here the transverse energy $\left(E_{\mathrm{T}}\right)$ spectrum is shown in $200 \mathrm{GeV} / \mathrm{C} \mathrm{p}-\mathrm{pb}$ collisions as measured by the HELIOS collaboration at the SPS. This distribution is very broad (and therefore very interesting!), and not at all well reproduced by HIJET. The p-p spectrum shown in this figure is the same data as in Fig. 6 , scaled to the rapidity interval of the HELIOS experiment. Here, as seen in Fig. 6, the HIJET (ISAJET) result reproduces the data extremely well. 


\section{Nucleus-Nucleus Collisions}

There is very little data with which to evaluate the HIJET extrapolation from the p-nucleus case of nucleus-nucleus collisions. The comparisons which can be made are encouraging. Two examples are shown here. Figure 14 shows the pseudo-rapidity distribution for charged particles produced in collisions of $\alpha$-particles, accelerated in the CERN PS accelerator, with an emulsion target. Central collisions are selected on the basis of the number of grey tracks observed. The HIJET result, calculated for impact parameters less than .5 fermi, agrees quite well with both the shape (angular distribution) and area (total charged particle multiplicity) of the measured curve.

Figure 15 shows a well-known cosmic ray event observed by the JACEE collaboration (Phys. Rev. Lett. 50, 2062). The pseudo-rapidity distribution is plotted for the 1015 charged particles produced in this collision, for which the projectile was identified as a silicon nucleus with an energy of about $5000 \mathrm{GeV} / \mathrm{amu}$. The HIJET result shown is an average over 50 such collisions, with impact parameters less than .5 fermi. The mean number of charged particles per event in this sample is 946 , and it is evident that these HIJET events are very similar to the observed event. 


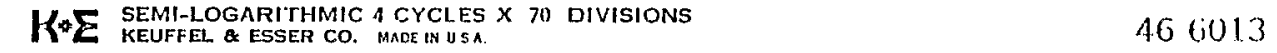

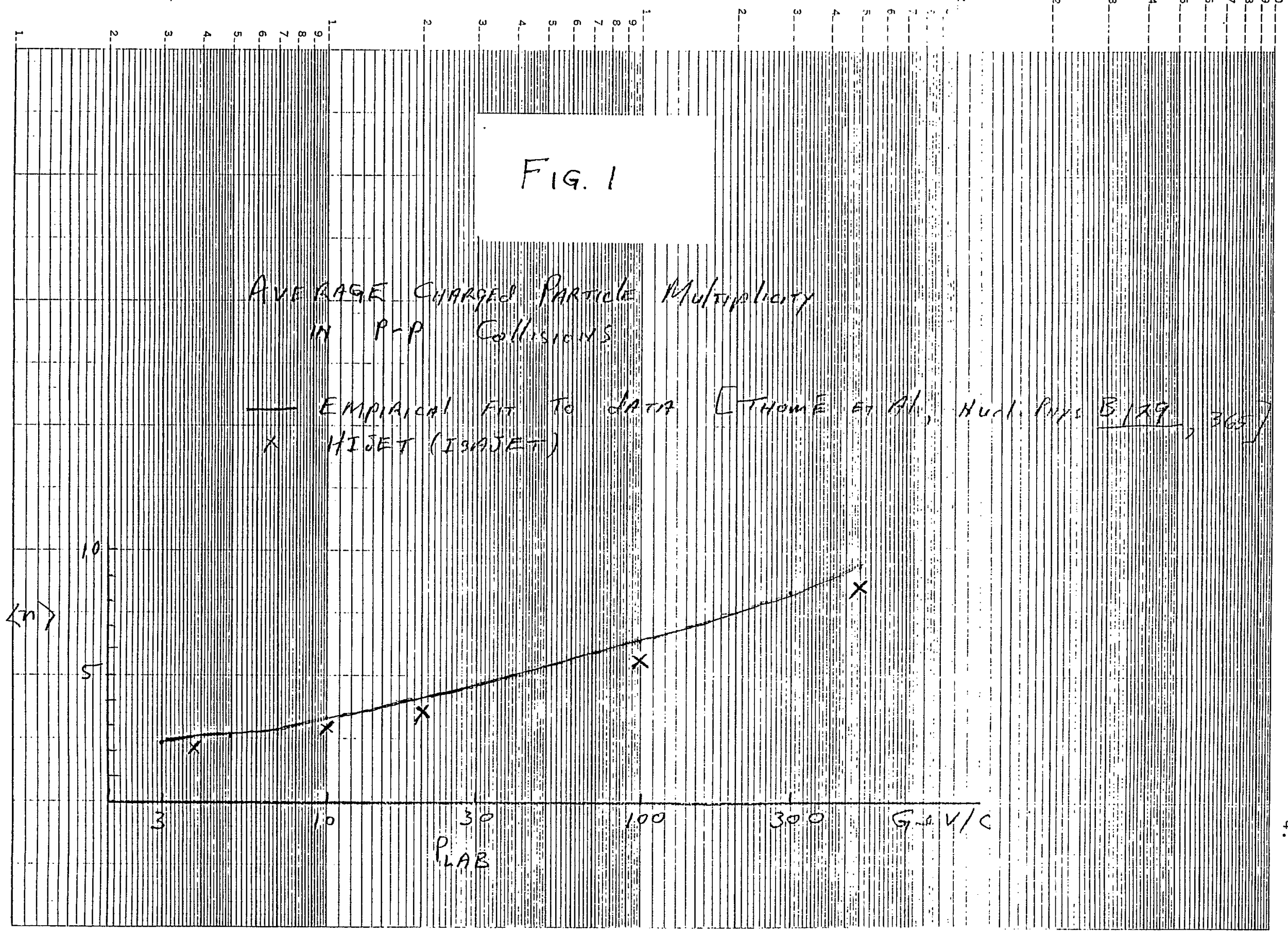




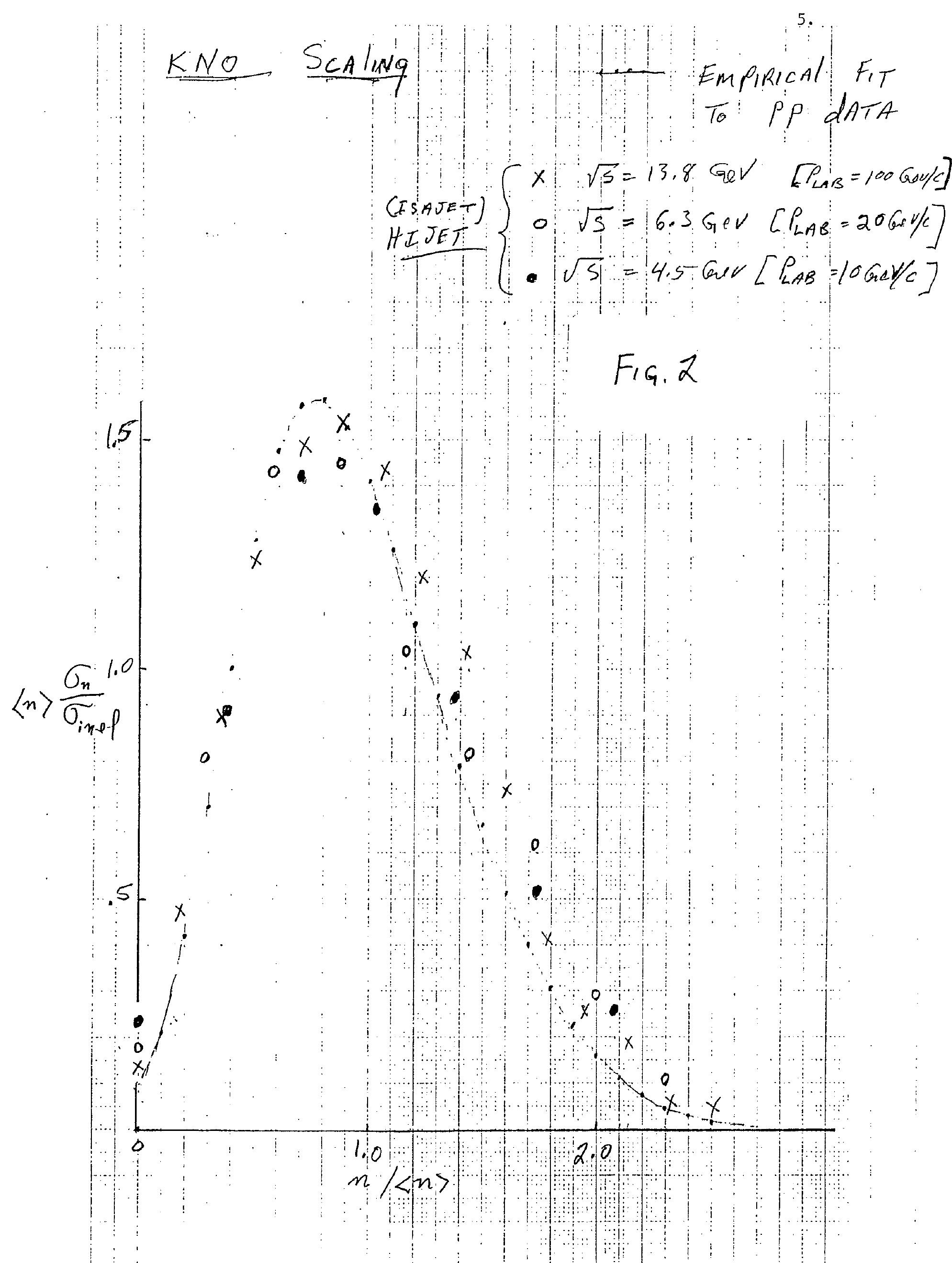




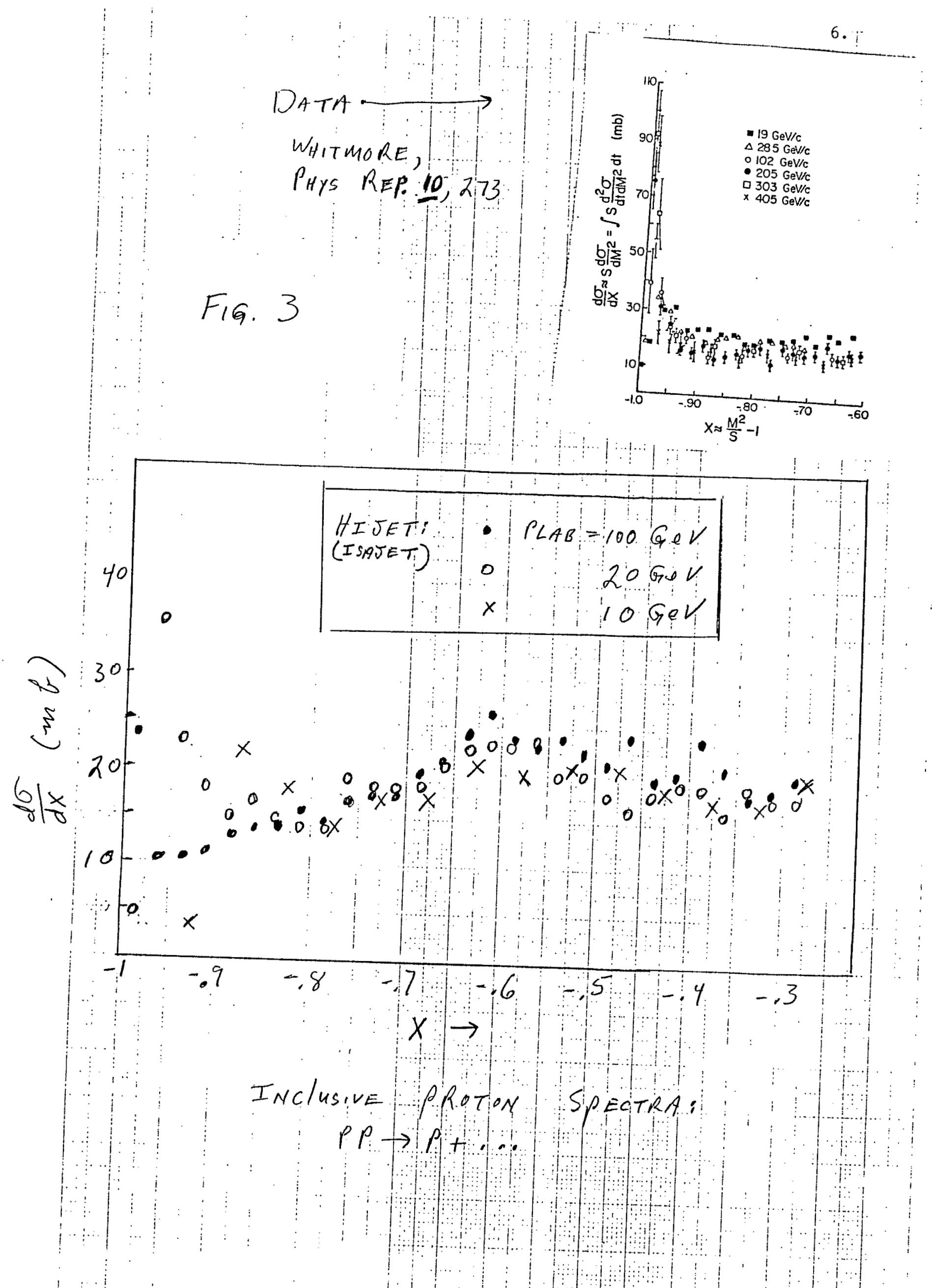


7.

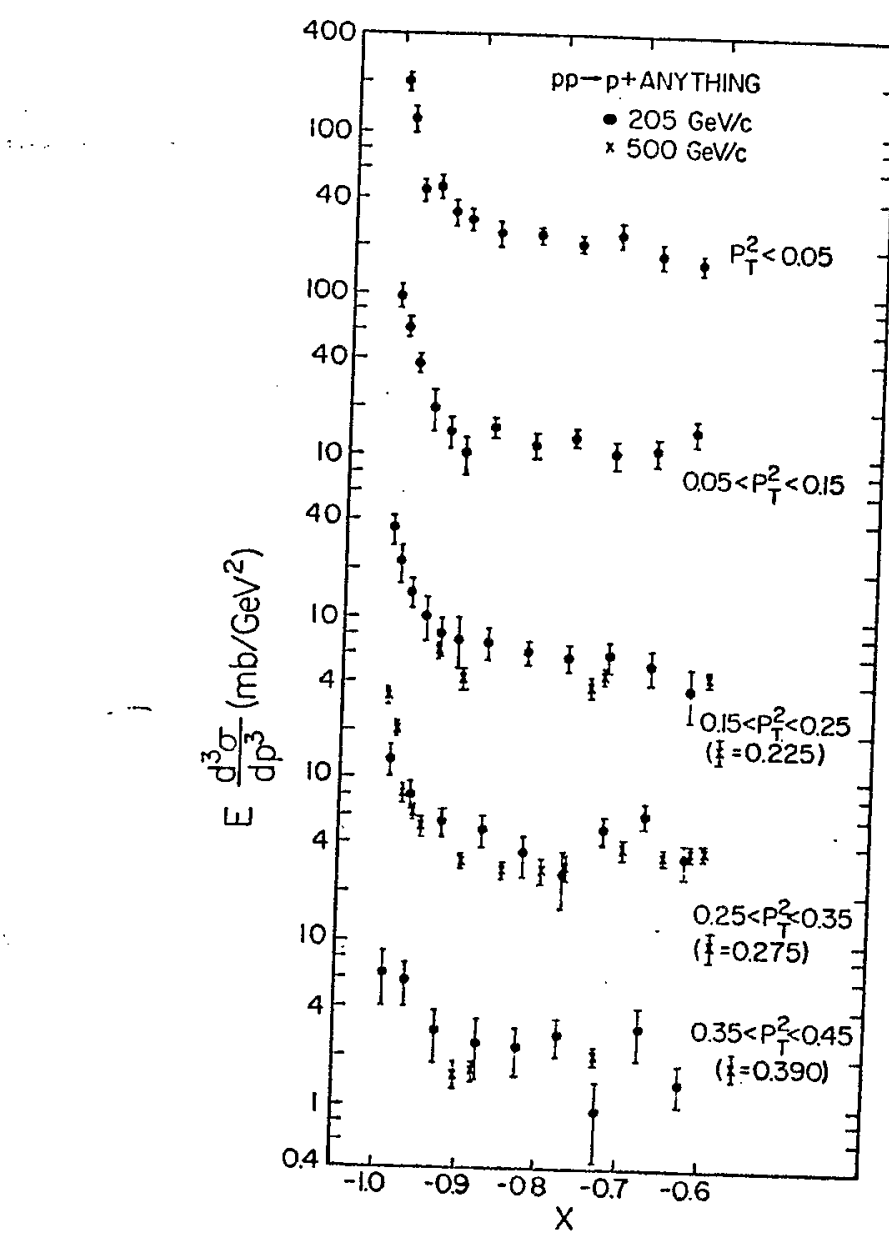

Fig. 50 Invariant cross sections for the reaction $\mathrm{pp} \rightarrow \mathrm{p}+$ anything as a function of $x=2 p_{L} / \sqrt{s}$ for fixed values of the trans verse momentum.

J. WHIT MORE, PHyS. REP. 10, 273 (1974)

Fig. $4 a$ 


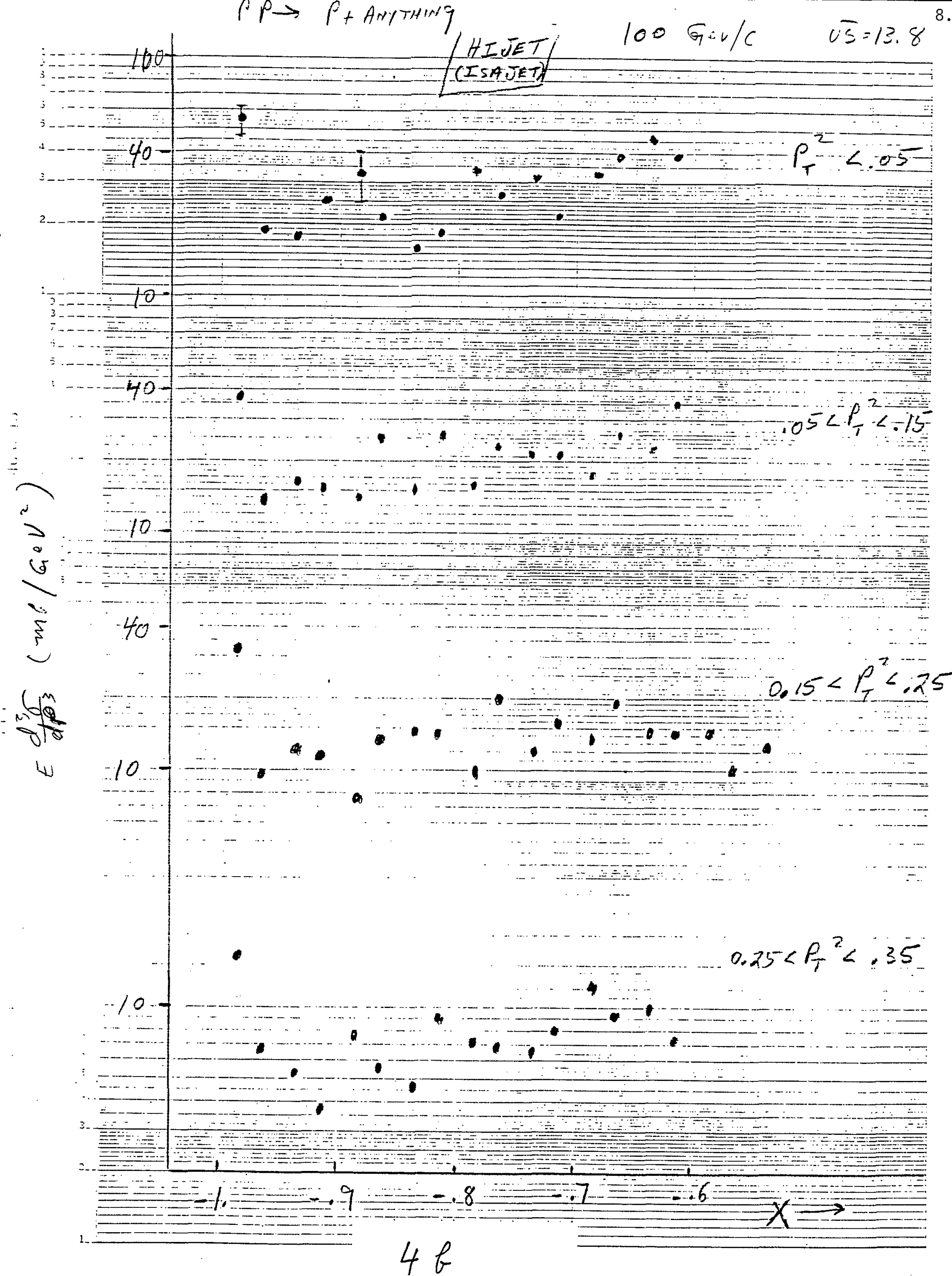


9

$F_{1 G} .5$

$200 \mathrm{GeV} / \mathrm{C} \mathrm{PP}$

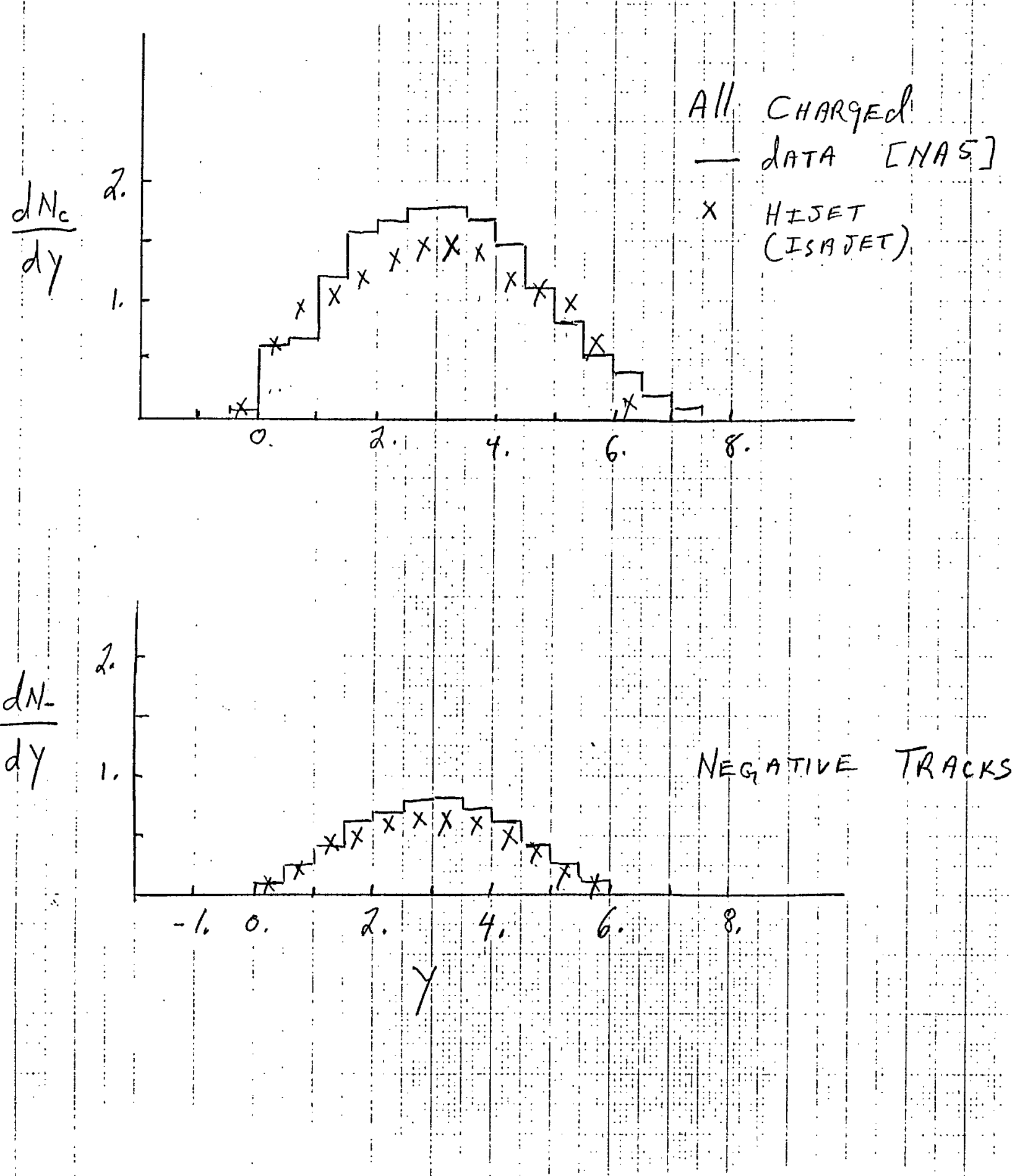


$P=A:$ CHARGEd $:$ PARTIClE: Multiplicity i1.

$\triangle$ : CERN SPSS (dE MARZO ET Al.)

- FERmilab (Elins ET Ali)

$X$ HIJET
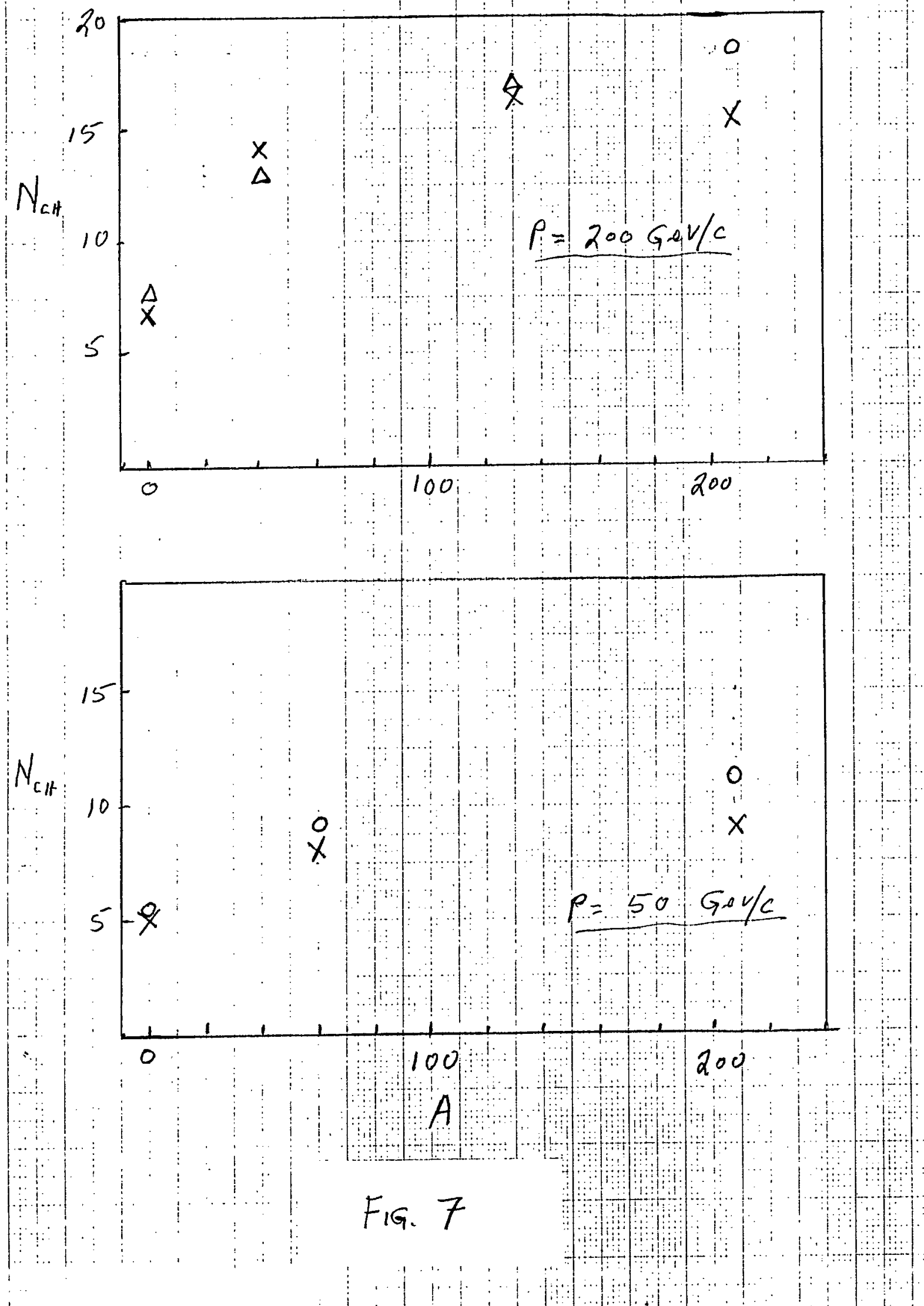


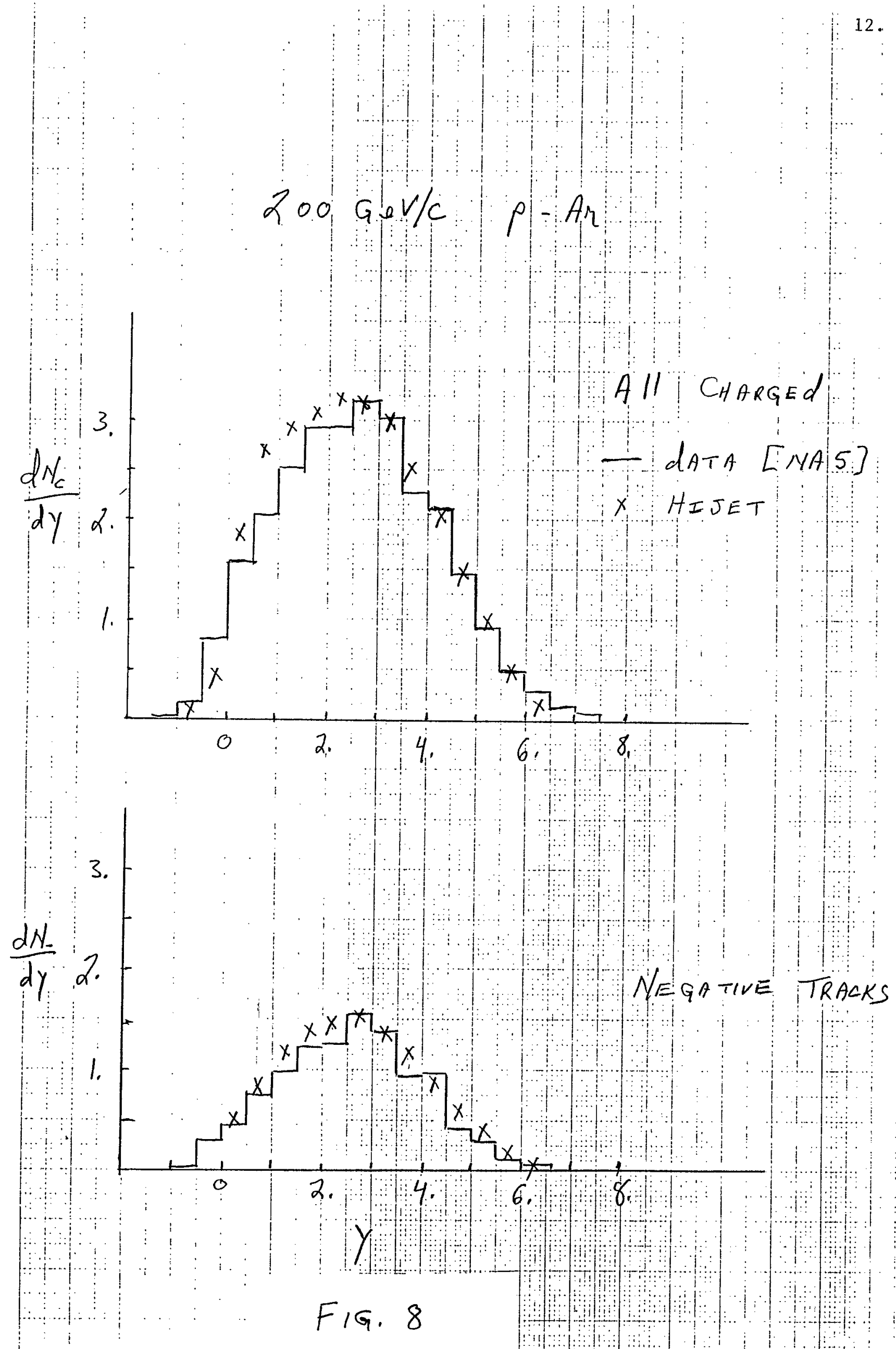




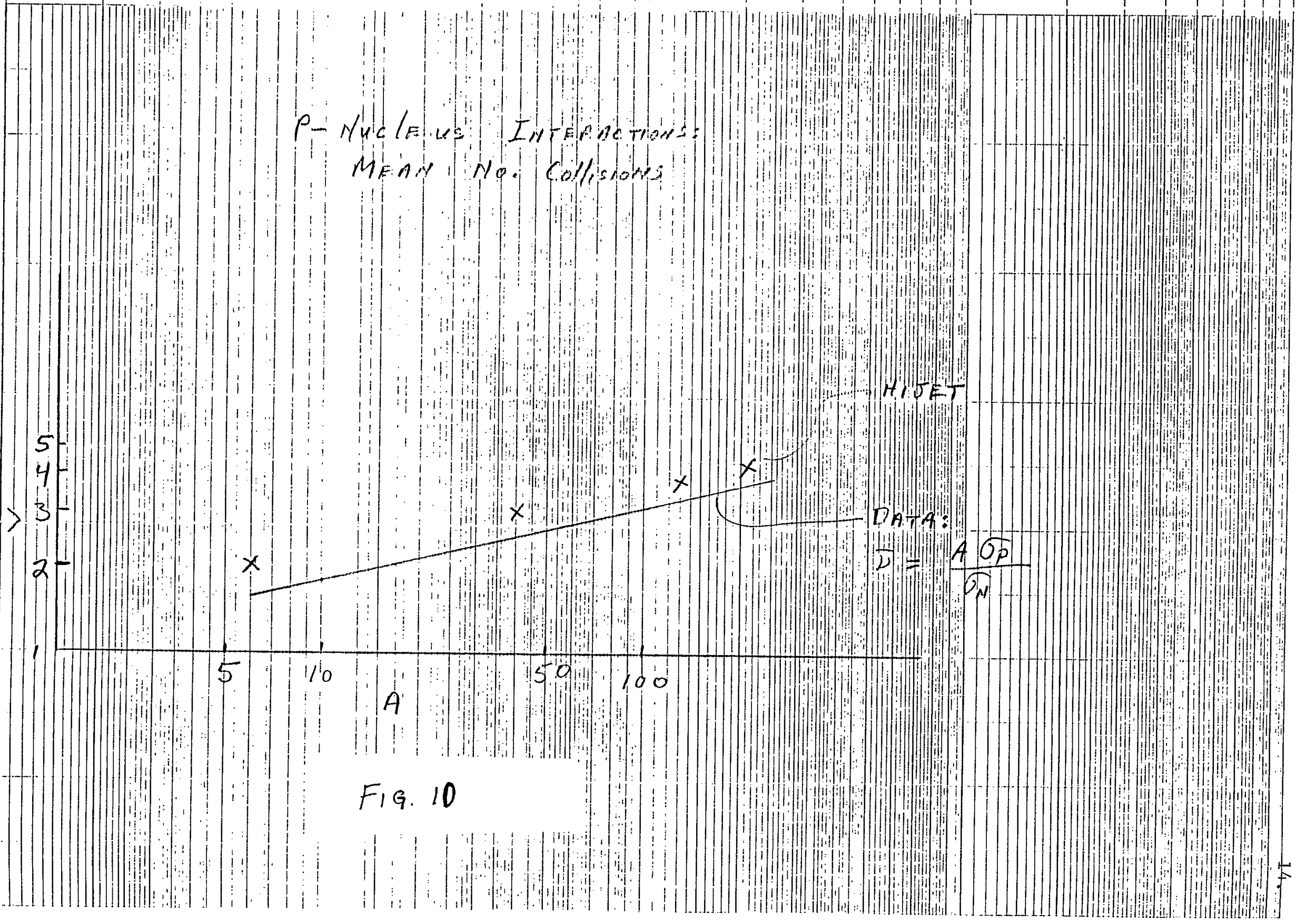




$$
70 \text { Livistorss }
$$

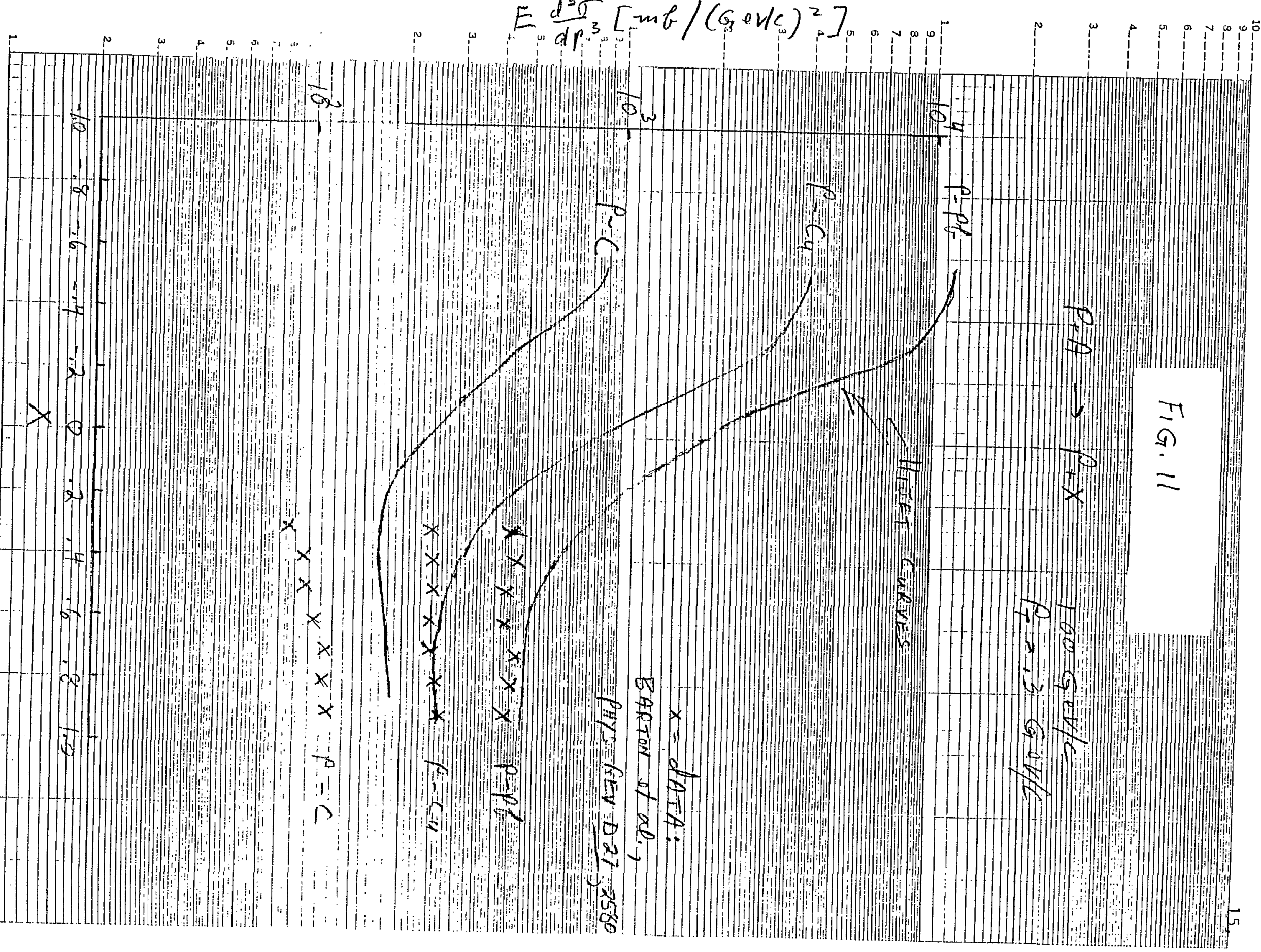




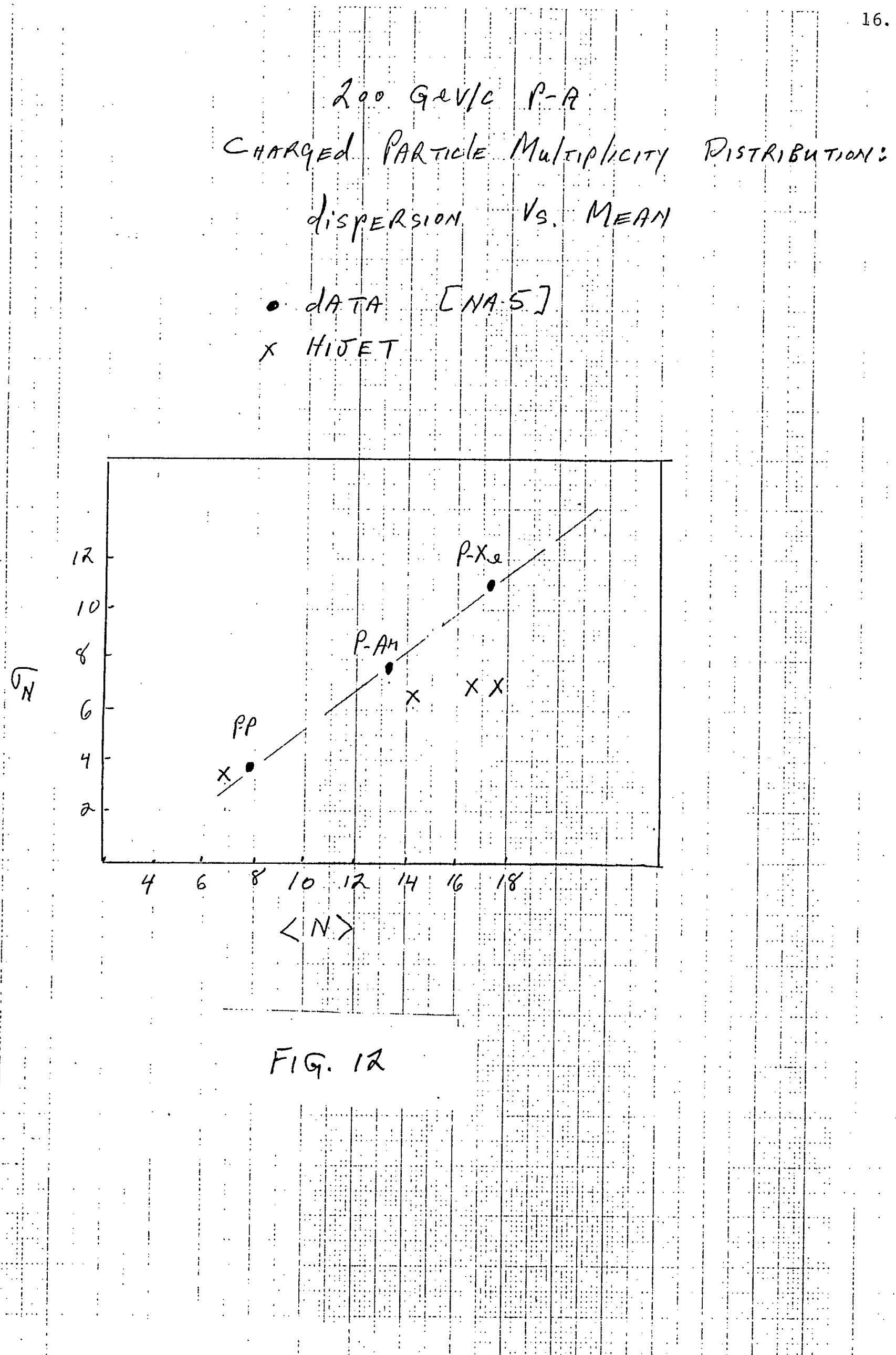




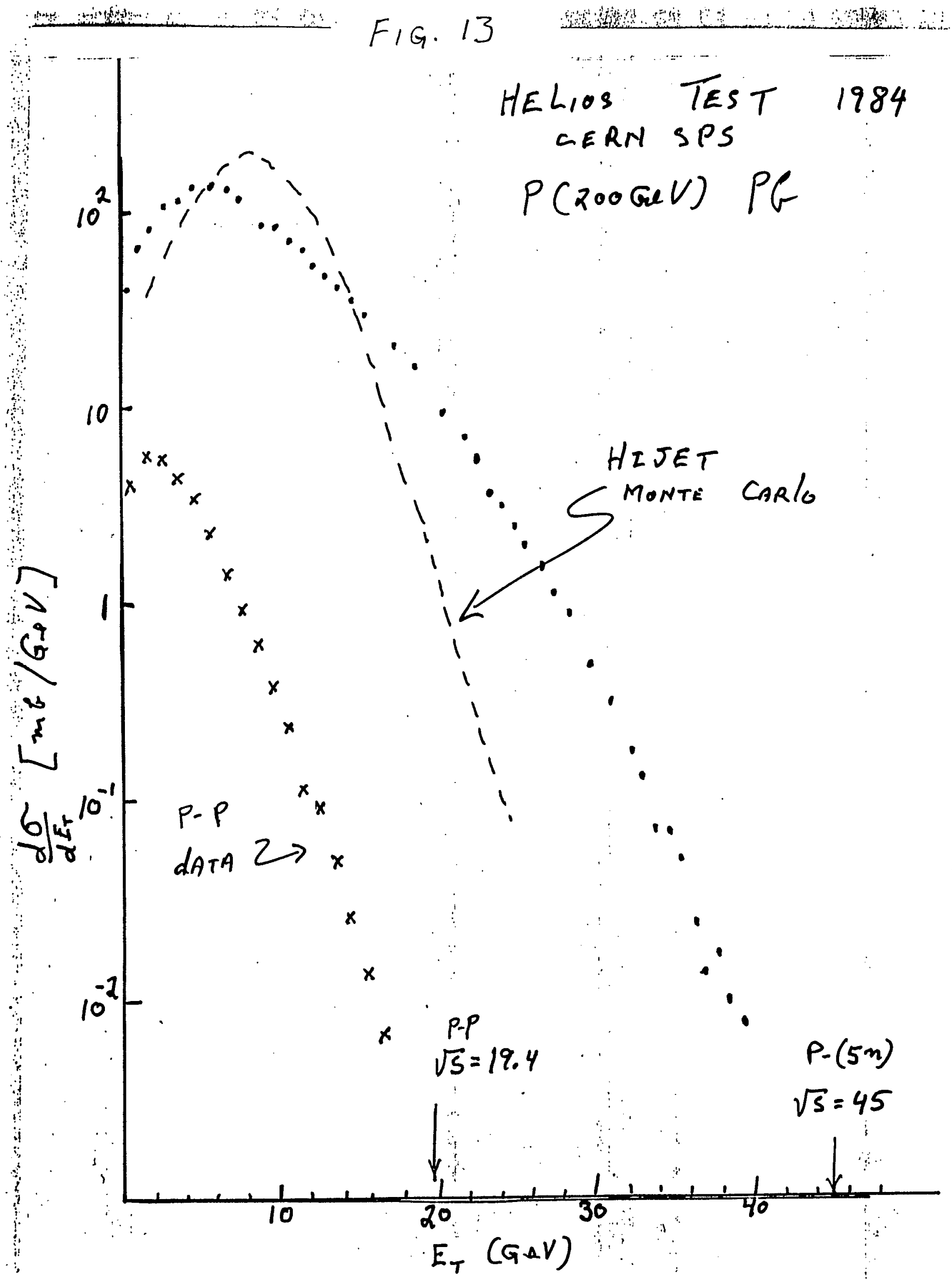


JACEE Collab.

$$
\sqrt{5} / A=
$$

$5:+$ Emulsion $\left(A g B_{n}\right)$

$50+50 \mathrm{GeV} /$ mucleon

1015 clianged $\quad 300$

${ }^{300} \int-\operatorname{MCM}(5 \mathrm{TeV} / \mathrm{n})$

-. WNM(5 TeV/n)

$$
\begin{aligned}
& \text { * H HIJET } s_{i}+A g \\
& \text { AUERAQE OUER } 50 \\
& \text { CENTRAC CollisioNs } \\
& \left\langle\eta_{C H}\right\rangle=946
\end{aligned}
$$

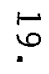

Fig. 15

: 兄 